\title{
1 Problemstellung, Auftrag und Zielsetzung
}

Zielsetzung der Nutzenbewertung ist die Darlegung eines kausalen Zusammenhangs zwischen Intervention und positivem oder negativem Effekt für den Patienten. Dieser Wirksamkeitsnachweis ist Gegenstand der evidenzbasierten Medizin. Gesundheit ist ein multidimensionales Konstrukt und wird in klinischen und nicht-klinischen Effektparametern gemessen. Patientenrelevante Zielgrößen in der Bewertung von Arzneimitteln sind gemäß $\$ 35 \mathrm{a}$ SGB V die Mortalität, die Morbidität und die Lebensqualität. Als mögliche Nutzendimensionen des Patientennutzens sollen insbesondere die Verbesserung des Gesundheitszustandes, Verkürzung der Krankheitsdauer, Verlängerung der Lebensdauer, Verringerung der Nebenwirkung und die Verbesserung der Lebensqualität berücksichtigt werden ( $\$ 35 \mathrm{~b}$ SGB V). Diese Dimensionen könnten auch auf Medizinprodukte übertragen werden. Endpunkte sollten zuverlässig und korrekt Änderungen des Gesundheitszustandes abbilden.

\subsection{Kritik an der Zulassungspraxis}

Der Weltmarkt für Medizintechnologien belief sich im Jahr 2012 auf ca. 220 Milliarden Euro. Allein der europäische Markt für Medizintechnologien wird dabei auf 70 Milliarden Euro geschätzt. ${ }^{[3]}$ Davon entfallen ca. 22 Milliarden Euro auf den deutschen Markt. ${ }^{[3]}$ In dieser Branche arbeiten in Deutschland ca. 190.000 Menschen in über 12.00o Unternehmen. ${ }^{[3]}$ Angesichts des immensen Fortschritts und des immer schneller wachsenden Anteils von innovativen Medizinprodukten (MP) hat der Markt für MP eine wichtige ökonomische Bedeutung. ${ }^{[4]}$ Der deutsche Markt ist hinter den USA und Japan der drittgrößte Medizintechnik-Markt der Welt. ${ }^{[3]}$ 
Innovative MP sind imstande, Untersuchungs- und Behandlungsmethoden zu verbessern. Wenn MP jedoch Mängel aufweisen, stellen sie eine Gefährdung für den Patienten dar. ${ }^{\left[{ }^{5]}\right.}$ Kritisch diskutiert wurde die SAMMPRIS-Studie (Stenting and Aggressive Medical Management for Preventing Recurrent Stroke in Intracranial Stenosis), welche dokumentierte, dass bei der Behandlung von intrakraniellen Stenosen mit Wingspan-Stents das Risiko für Schlaganfälle und Todesfälle zweieinhalb Mal höher war, als bei der intensiven medikamentösen Therapie. ${ }^{[6]}$ Zudem warnten Experten bei Metall-auf-Metall-Hüftprothesen davor, dass es zu gesundheitsschädlichem Metallabrieb kommen könnte. [7, ${ }^{8}$ In den letzten Jahren wurden daneben unter anderem ein undichtes Lungenversiegelungssystem $\left(\right.$ PleuraSeal $^{\circledR}$ ) und die Elektrode Sprint Fidelis ${ }^{\circledR}$ vom Markt genommen. ${ }^{[7,9,10]}$ Diese Vorkommnisse lösten weltweit Reaktionen bei Regulierungsbehörden aus. Die Kritik an den Markteintrittsregelungen von MP wurden heftiger ${ }^{[1]}$, als aufgrund krimineller Machenschaften des französischen Herstellers von Brustimplantaten PIP (Poly Implant Prothèse) statt medizinischem, nichtzulässiges Silikon für die Herstellung des Implantates verwendet wurde. ${ }^{[12]}$ Aufgrund der Ereignisse ${ }^{[3-15]}$ sah man sich auf europäischer Ebene veranlasst, die bestehenden Kriterien für die Zulassung vor dem Inverkehrbringen und die Überwachung nach dem Inverkehrbringen ${ }^{[16]}$ zu ändern. ${ }^{[7]]}$ Kritiker des europäischen Zulassungssystems verweisen darauf, dass im Vergleich zu den USA der europaweite Marktzugang relativ früh erfolgt. $[9,10,18]$

In Deutschland wurde in diesem Zusammenhang von Seiten des Instituts für Qualität und Wirtschaftlichkeit im Gesundheitswesen (IQWiG) kritisiert, dass der patientenrelevante Nutzen bisher weder beim Marktzugang noch in $\mathrm{Zu}$ sammenhang mit der Erstattungsfähigkeit systematisch geprüft wird. Aus diesem Grund sprach man sich dafür aus, dass die Prozesse für MP mit „mittlerem oder hohem Risikopotenzial und solche mit relevantem ökonomischen Mehraufwand grundlegend neu reguliert und strukturiert werden" sollen. ${ }^{[9]}$ Das Bundesministerium für Gesundheit (BMG) hat am 17. Dezember 2014 einen ersten Regierungsentwurf für ein „Gesetz zur Stärkung der Versorgung in der gesetzlichen Krankenversicherung“ (GKV-VSG) vorgelegt. Der Bundestag hat dieses Gesetz am 11. Juni 2015 beschlossen. Das Gesetz regelt für eine eng definierte Gruppe von NUB die Durchführung einer Nutzenbewertung und damit für diese die Erstattungsfähigkeit durch die GKV. Der G-BA hat damit zukünftig die gesetzliche Aufgabe, für MP der Klassen IIb und III mit neuem theoretisch-wissenschaftlichen Konzept und besonders invasivem Charakter eine Nutzenbewertung durchzuführen. Die Nutzenbewertung wird parallel zu einem NUB-Entgeltantrag nach $\mathbb{} 6$ Absatz 2 Satz 3 des Krankenhausentgeltgesetzes initiiert.

Nach Inkrafttreten des Gesetzes sind die anfragenden Krankenhäuser erstmals verpflichtet, dem Gemeinsamen Bundesausschuss Informationen über den Stand der wissenschaftlichen Erkenntnisse zu Methoden mit neuem theoretisch-wissenschaftlichem Konzept sowie zu der Anwendung des MP vorzulegen. Die Übermittlung der Unterlagen erfolgt im Benehmen mit dem Herstel- 
ler derjenigen MP der Klassen IIb und III, die im NUB-antragstellenden Krankenhaus bei der Methode zur Anwendung kommen sollen. Die Organe der Selbstverwaltung stehen vor der Aufgabe, die Leistungsfähigkeit durch die Abwägung des Nutzens und Schadens (bzw. des Nutzen- und Schadenspotenzials) zu ermitteln. Die Leistungsfähigkeit wird durch das Potenzial und den Nutzen dokumentiert.

Der Nutzen wird aus multiplen Zielkriterien bzw. patientenrelevanten Endpunkten abgeleitet. Die aktuelle Diskussion über die gesetzlichen Änderungen sowie die Aufgabendefinitionen des G-BA und IQWiG im Gesundheitswesen stärken das Konzept der evidenzbasierten Medizin (EbM) als Crundvoraussetzung für eine am Patientennutzen ausgerichtete Gesundheitsversorgung. ${ }^{[19]}$

Durch das Messen der klinischen Effekte, das Bewerten der realisierten Erfolgsgrößen und eine Abwägung von Nutzen und Schaden soll eine Entscheidung über die Erstattungsfähigkeit der Methode vorgenommen werden. Das Bewertungsverfahren bzw. die rationale Entscheidung über den Marktzugang und die Erstattung soll die Qualität der Versorgung garantieren. Das Bewertungsverfahren wird im Gesetz oder als Verfahrensanweisung der zuständigen Behörden und Institutionen vorgegeben. Offen bleibt, wie diese regulatorischen Verfahren inhaltlich strukturiert und angewendet werden können.

\subsection{Fragestellungen des Gutachtens}

In Anbetracht der aktuellen Entwicklungen in Bezug auf die Einführung einer Nutzenbewertung für NUB mit neuen theoretisch-wissenschaftlichen Konzepten und MP der Klassen IIb und III sowie aktive MP im Rahmen des $\$ 137 \mathrm{~h} \mathrm{SGB} \mathrm{V} \mathrm{formuliert} \mathrm{dieser} \mathrm{Beitrag} \mathrm{die} \mathrm{Anforderungen} \mathrm{an} \mathrm{eine} \mathrm{sachadäqua-}$ te Nutzenbewertung von Methoden mit MP höherer Klassen. Im Rahmen dieses Gutachtens soll verschiedenen Fragestellungen nachgegangen werden:

- Wie kann ein sachadäquates Verfahren der Nutzenbewertung für nichtmedikamentöse Methoden mit MP umgesetzt werden?

- Wie kann die klinische Erfolgsmessung von neuen theoretisch-wissenschaftlichen Konzepten umgesetzt werden?

- Wie können die Patienten bei der Bewertung der gemessenen klinischen Studienergebnisse ausreichend berücksichtigt werden?

- Wie kann der aggregierte Gesamtnutzen, basierend auf multiplen klinischen und nicht-klinischen Endpunkten, aggregiert werden?

- Gibt es internationale Erfahrungen zu effizienten Bewertungsverfahren, welche die Abwägung von zeitkritischem Zugang und Sicherheit optimal unterstützen? 
Im Rahmen dieses Gutachtens erfolgt die

- Dokumentation der gesetzlichen Voraussetzungen in Verbindung mit den Rahmenbedingungen aus dem GKV-Versorgungsstärkungsgesetz.

- Definition der Problemstellung der Nutzenbewertung von NUB.

- Dokumentation der Konzepte der Nutzenbewertung, unter anderem auf Basis des IQWiG- Methodenpapieres.

- Dokumentation der Anforderungen an die Prozesse des Messens, Bewertens und Entscheidens.

- Diskussion der methodischen Grundlagen in Bezug auf neue theoretischwissenschaftliche Konzepte von NUB mit MP hoher Klassen.

- Dokumentation von möglichen Lösungsansätzen im Kontext der adaptiven Nutzenbewertung und bedingten Erstattung.

\subsection{Zielsetzung des Gutachtens}

Mit der Einführung des $\mathbb{1} 137 \mathrm{~h}$ SGB V wird die Nutzenbewertung von NUB mit MP mit einem hohen Risikopotenzial neu strukturiert. Diese Publikation fokussiert die methodischen Herausforderungen bei der Nutzenbewertung von innovativen Untersuchungs- und Behandlungsmethoden.

Vor diesem Hintergrund verfolgt dieses Gutachten drei Ziele:

1. Diskussion des Messens von klinischen und nicht-klinischen Erfolgskriterien

Das Messen stellt eine wesentliche Herausforderung im Nutzenbewertungsverfahren dar. Die Entscheider stellen zunehmend höhere Anforderungen an den Evidenzgrad, wonach der Goldstandard des wissenschaftlichen Nachweises der Kausalität von Effekten mittels randomisierter kontrollierter Studien (RCT) erbracht werden sollte. Dies ist jedoch dann problembehaftet, wenn die Umsetzung von konventionellen Studiendesigns zu komplex, zu zeitintensiv oder schlichtweg unmöglich ist. Selbst wenn Effekte objektiv nach allen Anforderungen an eine RCT gemessen werden, stehen diese Daten nicht immer in einer Beziehung zu den in der Realität zu beobachtenden Ergebnissen. Zudem sind RCTDaten für MP in den Entscheidungsprozessen nicht immer verfügbar. Die Gestaltung und Umsetzung von konventionellen Studien ist teilweise schwierig. Einen Lösungsansatz stellen adaptive Studiendesigns dar. Ein adaptives Design kann als (klinische) Studie definiert werden, die prospektiv geplante Änderungen basierend auf der Akkumulation von Studiendaten erlaubt, ohne dabei die Integrität und Validität der Studie zu verletzen. ${ }^{[20]}$ Adaptive Designs eröffnen damit die Möglichkeit, erste Studienergebnisse zu bestimmten Zeitpunkten während der Durchführung auszuwerten und das Studiendesign entsprechend anzupassen. ${ }^{[2]}$ Wenn sie richtig umgesetzt werden, können adaptive Designs demnach den Ressourcenbedarf reduzieren und/oder die Chance auf den Studienerfolg erhöhen. ${ }^{[20]}$ 


\section{Diskussion multikriterieller Entscheidungsprobleme und Patientenpräferenzen}

Nahezu jede Aktivität im Gesundheitswesen basiert auf der Bewertung von Alternativen. Alternativen werden oft durch mehrere Eigenschaften beschrieben. Neue Methoden können nur durch multiple Zielkriterien beschrieben und gegeneinander abgegrenzt werden. Auf der regulatorischen Ebene beurteilen Entscheidungsträger multiple klinische oder nicht-klinische Endpunkte, um den Erfolg oder Misserfolg einer Maßnahme zu beurteilen bzw. über deren Erstattungsfähigkeit zu entscheiden. Auf klinischer Ebene bewerten Ärzte Handlungsoptionen für die Therapie von Patienten innerhalb der von politischen Entscheidungsträgern festgesetzten Zielkriterien. Schließlich bewerten Patienten die ihnen vorgelegten Behandlungsalternativen im Bestreben ihr allgemeines Wohlbefinden zu erhöhen. ${ }^{[2]}$ Eine Grundidee bei der Bewertung des Patientennutzens ist, dass sich das Beurteilungsobjekt durch ein oder mehrere Eigenschaften (Attribute oder Beurteilungskriterien) beschreiben lässt. ${ }^{[22,23]}$ Damit werden Beurteilungsobjekte als Bündel von Eigenschaften definiert. ${ }^{[24]}$ Bei der Nutzenbewertung werden damit nicht mehr nur klinische oder nicht-klinische Erfolgsparameter (z.B. Laborparameter) gemessen, sondern auch der Nutzen und Schaden gegeneinander auf Basis von Werturteilen oder Patientenpräferenzen abgewogen. ${ }^{[25]}$ International wird die multikriterielle Entscheidungsanalyse (Multi-Criteria Decision Analysis, MCDA) als möglicher Lösungsansatz diskutiert. Dabei beschreibt MCDA als Oberbegriff verschiedene analytische Methoden zur Berücksichtigung multipler Entscheidungskriterien. ${ }^{[26,27]}$ Allen Ansätzen ist gemein, dass der Patientennutzen (Gesamtnutzen) als multidimensionales Konstrukt angesehen wird, welcher nur unter Einbeziehung der betroffenen Patienten oder weiterer Stakeholder bewertet werden kann. MCDA kann damit eingesetzt werden, um komplexe Entscheidungen über neue theoretisch-wissenschaftliche Konzepte zu unterstützen und die Partizipation von Patienten zu fördern. [28]

\section{Diskussion der Abwägung von zeitkritischem Zugang und bestmöglichem Evidenzgrad}

Die Entscheidung über die Erstattungsfähigkeit wird auf Basis der Informationen über den Gesamtnutzen getroffen. Eine rational begründete Entscheidung über MP basiert auf vorab definierten Zielen und transparenten Werturteilen. Die Entscheidungskriterien können von den Zielen der Effektivität und der Wirtschaftlichkeit eines MP abgeleitet werden. Gegenstand der Entscheidung können die Innovationen und Vergleichstherapien in einem Indikationsgebiet sein (indikationsspezifische Entscheidung). Denkbar, aber in Deutschland aktuell nicht angewandt, ist auch eine Entscheidung über alle Indikationsgebiete (indikationsübergreifende Entscheidung). Vor dem Hintergrund des GKVVersorgungsstärkungsgesetzes lassen sich für die Ausgestaltung der Nutzenbewertung von NUB mit MP hoher Sicherheitsklasse unterschiedliche Vorgehensweisen ableiten. Vor dem Hintergrund des zunehmenden 
Drucks der Patienten auf den rechtzeitigen bzw. zeitkritischen Zugang zu innovativen Behandlungsmethoden und MP, vor allem bei Indikationen mit ungedecktem Handlungsbedarf, müssen Ansätze für einen sicheren und qualitativ hochwertigen, aber zugleich zeitnahen Zugang geschaffen werden. Die adaptiven Pfade kommen ursprünglich aus der Zulassung von Arzneimitteln für seltene Erkrankungen. Dieser Ansatz wird aktuell sowohl von der EMA ${ }^{[2]}$, dem National Institute for Health and Care Excellence (NICE) und der Food and Drug Administration (FDA) genutzt. ${ }^{\left[{ }^{\circ 0}\right]}$ Die adaptiven Pfade ermöglichen einen schnelleren Zugang von Patienten zu innovativen Therapien. Dabei ist jedoch zu beachten, dass in diesem Verfahren sowohl die Patienten, Leistungserbringer, Hersteller als auch Aufsichtsbehörden bereit sein müssen, höhere Risiken und Unsicherheiten in Bezug auf den Nachweis von Wirksamkeit und Sicherheit zu akzeptieren. ${ }^{[2,30]}$ Ausgehend vom bisherigen Status Quo in Deutschland könnten diese adaptiven Pfade einen signifikanten Beitrag zur Patientensicherheit leisten. Bezogen auf die Nutzenbewertung von NUB mit MP hoher Klassen stellen adaptive Pfade gewissermaßen eine verlängerte Erprobungsregelung dar. NUB mit MP hoher Klassen würden zunächst „vorläufig“ bewertet und ein entsprechender Nutzen zuerkannt. Über die explizite Höhe des zusätzlichen Nutzens im Vergleich zu bereits etablierten Verfahren würde über einen definierten Zeitraum mittels umfangreicherer Studien entschieden. Einen ähnlichen Prozess beschreibt auch die neue Regelung des $\mathbb{1} 137 \mathrm{~h} \mathrm{SGB} \mathrm{V}$.

Das Gutachten formuliert die Anforderungen an eine sachadäquate Nutzenbewertung von Methoden mit MP höherer Klassen auf Basis des IQWiG-Methodenberichtes $4.2^{[1]}$, der neuen Gesetzgebung und unter Berücksichtigung der internationalen Erfahrungen sowie der Besonderheiten von MP hoher Klassen. 